EPHOU 02-009

December 2002

\title{
Mean-field Approach to the Derivation of Baryon Superpotential from Matrix Model
}

\author{
Hisao Suzuki \\ Department of Physics, \\ Hokkaido University \\ Sapporo, Hokkaido 060 Japan \\ hsuzuki@phys.sci.hokudai.ac.jp
}

\begin{abstract}
We discuss how to obtain the superpotential of the baryons and mesons for $S U(N)$ gauge theories with $N$ flavour t
\end{abstract}




\section{Introduction}

Recently, Dijkgraaf and Vafa [1, 2, 3] proposed a general prescription for computing effective glueball superpotentials via planar diagrams of matrix theories. Several tests have been achieved for this conjecture [4- [1] for the fields in adjoint representations. A recent paper [12, 13] proved a correspondence for matter fields in the adjoint representation. A natural extension of the construction is to the theories with fields in the fundamental representations. The gauge theory effective potential for glueball superfield $S$ is given by

$$
W_{D V}\left(S, \Lambda, \lambda_{a}\right)=N_{c} S\left(-\log \left(S / \Lambda^{3}\right)+1\right)+N_{c} \frac{\partial \mathcal{F}_{\chi=2}\left(S, \lambda_{a}\right)}{\partial S}+\mathcal{F}_{\chi=1}\left(S, \lambda_{a}\right)
$$

where $\mathcal{F}_{\chi=2}$ is the contribution from adjoint fields and $\mathcal{F}_{\chi=1}\left(S, \lambda_{a}\right)$ is the contribution from the fields in the fundamental representations. The programm was successfully used to compare matrix model results with known superpotentials of meson fields [14]-[21].

The advantage the matrix theory should be that we can prove some basic hypothesis or conjectures which cannot be provided by the standard field theoretical methods. In fact, a derivation of the non-renormalization theorem was performed in [22].

One of the biggest problems is the inclusion of the baryonic fields. Matrix theory may be useful to get insights for the baryons. This problem was treated in a recent papers [23, 24]. The paper [23] showed that the first order calculation in the perturbative technique agrees with the field theoretical result. In [24], they showed that for $S U(2)$ gauge fields with 2 flavor models, we can obtain superpotential for baryonic fields. In this model, the baryons can be expressed by bilinear fields and the matrix integration can be performed. However, for $S U(N), N>2$, the baryons are composed of more than trilinear fields so that we cannot easily perform the matrix integrations.

In this paper, we consider the theories with $S U(N)$ gauge fields with $N$ flavour. The quantum moduli space is conjectured to be parametrized by meson fields and baryons by [26, 27, 28]

$$
\operatorname{det} M-B \tilde{B}=\Lambda^{2 N}
$$


Our aim of this paper is the derivation of this constraint from the matrix model. Since baryonic perturbation is complicated, we will use the mean-field method to estimate the superpotential, which is related to the free energy of the matrix model. It will turn out that the self-consistency equation for the mean field depends on $N$. We will therefore assume a planar limit of the equation. With this assumption, we will show that we can get a potential which almost agrees with the expected results. Before doing this, we will first treat the field theoretical result which should be compared to the matrix theory result.

\section{Effective Superpotentials for $N_{c}=N_{f}$}

We will first argue how to obtain effective superpotential from the field theory. We consider $S U(N)$ gauge theories with $N$ quark flavors $Q_{a}^{i}$ and $\tilde{Q}_{i}^{a}$. We diagonalize the mass matrix and consider the tree level superpotential

$$
W_{\text {tree }}(Q, \tilde{Q})=m_{i} \tilde{Q}_{i}^{a} Q_{a}^{i}+b \operatorname{det} Q+\tilde{b} \operatorname{det} \tilde{Q}
$$

which can be written in terms of meson operators and baryon operators

$$
M_{i}^{j}=Q_{i}^{a} \tilde{Q}_{a}^{j}, \quad B=\operatorname{det} Q, \quad \tilde{B}=\operatorname{det} \tilde{Q}
$$

as

$$
W_{\text {tree }}\left(X_{i}, B, \tilde{B}\right)=m_{i} X_{i}+b B+\tilde{b} \tilde{B}
$$

where we have defined $X_{i}=M_{i}^{i}$. In order to obtain the non perturbative part of the effective superpotential, we start with the Veneziano-Yankielowicz superpotential [25].

$$
W=N S-S \ln \frac{S^{N}}{\tilde{\Lambda}^{3 N}} .
$$

For $N$-flavour, we assume the matching relation $\tilde{\Lambda}^{3 N}=m_{1} m_{2} \cdots m_{N} \Lambda^{2 N}$. When we integrated in $X_{i}$ fields as $X_{i}=\partial_{m_{i}} W$, we get the following superpotential

$$
W=S \ln \frac{\Lambda^{2 N}}{X_{1} X_{2} \cdots X_{N}}+m_{i} X^{i},
$$


which can be promoted to

$$
W=S \ln \frac{\Lambda^{2 N}}{\operatorname{det} M}+m_{i} X^{i}
$$

If we include the baryon fields, it is natural to expect that the effective potential is given by

$$
W=S \ln \frac{\Lambda^{2 N}}{-B \tilde{B}+\operatorname{det} M}+b B+\tilde{b} \tilde{B}+m_{i} X^{i} .
$$

The equation $\partial_{S} W=0$ implies the constraint

$$
\operatorname{det} M-B \tilde{B}=\Lambda^{2 N},
$$

which is a desired result [26, 27, 28]. We assume that this potential is exact although the proof of the non-renormalization theorem of baryoionic perturbation may be very subtle.

To compare this result with the superpotential from the matrix models, we are going to integrated out the fields $B, \tilde{B}$ and $M_{i}^{j}$. Let us first denote

$$
\Delta=\operatorname{det} M-B \tilde{B}
$$

The equation $\partial_{B} W=0, \partial_{\tilde{B}} W=0$ implies

$$
B=-\frac{\Delta \tilde{b}}{S}, \tilde{B}=-\frac{\Delta b}{S} .
$$

The variation with respect to $M_{i}^{j}$ leads to

$$
\begin{aligned}
M_{i}^{j} & =0,(i \neq j) \\
m_{i} X_{i} & =\left(\frac{\Delta}{S} m_{1} m_{2} \cdots m_{N}\right)^{\frac{1}{N-1}} .
\end{aligned}
$$

From these relations, we find that $\Delta$ satisfies an equation

$$
\Delta=X_{1} X_{2} \cdots X_{N}-B \tilde{B}=\left(\frac{\Delta}{S}\right)^{\frac{N}{N-1}}-\left(\frac{\Delta}{S}\right)^{2} b \tilde{b} .
$$

when $b=0$, this equation has a solution $\Delta=S^{N} / m_{1} m_{2} \cdots m_{N}$. Therefore, we define a new variable by $\Delta=S^{N} / m_{1} m_{2} \cdots m_{N} \tilde{\Delta}$. Then the equation for $\tilde{\Delta}$ is given by

$$
\tilde{\Delta}=(1+\alpha \tilde{\Delta})^{N-1},
$$


where $\alpha$ is defined as

$$
\alpha=\frac{S^{N-2} b \tilde{b}}{m_{1} m_{2} \cdots m_{N}} .
$$

Substituting these variables in (2.7), we find that the effective superpotential can be written in the form

$$
W=N S\left(1-\ln S / \Lambda^{3}\right)+\mathcal{F}
$$

where $\mathcal{F}$ is

$$
\mathcal{F}=S \ln \frac{m_{1} m_{2} \cdots m_{N}}{\Lambda^{N}}-S \ln \tilde{\Delta}+(N-2) \alpha S \tilde{\Delta} .
$$

This is the final form of the superpotential which we want to compare in the next section. Note also that the superpotential (2.16) agrees with the one obtained in ref [23] although the derivation seems different.

\section{Mean-field Approximation for Matrix Model}

\subsection{Mean Field Approach}

Before discussing the model for baryonic perturbation, we will apply the mean-field method to the known models. The aim of doing this is to get a support for the validity of the mean-field method. The tree level superpotential we treat here is obtained by the deformation from $N=2$ SQCD theory with fundamental flavors $Q_{i}^{a}$ and $\tilde{Q}_{i}^{a}$ by adding a mass $M$ for the adjoint scalar $\Phi_{a}^{b}$ in $N=2$ vector multiplet

$$
W_{\text {tree }}(\Phi, Q, \tilde{Q})=\frac{1}{2} M \operatorname{Tr} \Phi^{2}+g \tilde{Q}_{i}^{a} \Phi_{a}^{b} Q_{b}^{i}+m \tilde{Q}_{i}^{a} Q_{a}^{i},
$$

where we have set the mass of the fundamental flavors to be $m_{1}=m_{2}=\ldots=m_{N_{f}}=m$ for simplicity. We consider the following free energy

$$
e^{-\frac{N}{S} \mathcal{F}}=\left(\frac{N \Lambda}{S}\right)^{N_{f}} \int d \Phi d Q d \tilde{Q} e^{-\frac{N}{S} W_{\text {tree }}(\Phi, Q, \tilde{Q})},
$$


Since the integral for the adjoint fields $\Phi_{a}^{b}$ is Gaussian, we can integrate out the fields and obtain

$$
e^{-\frac{N}{S} \mathcal{F}}=\left(\frac{N \Lambda}{S}\right)^{N_{f}} \int d \Phi d Q d \tilde{Q} e^{-\frac{N}{S}\left[m \tilde{Q}_{i}^{a} Q_{a}^{i}-\frac{g^{2}}{2 M} \tilde{Q}_{i}^{a} Q_{a}^{j} \tilde{Q}_{j}^{b} Q_{b}^{i}\right]}
$$

We re-scale the variables as

$$
Q_{i}^{a} \rightarrow\left(\frac{N}{S} m\right)^{-1 / 2} Q_{i}^{a}, \quad \tilde{Q}_{a}^{i} \rightarrow\left(\frac{N}{S} m\right)^{-1 / 2} \tilde{Q}_{a}^{i}
$$

to get

$$
e^{-\frac{N}{S} \mathcal{F}}=\left(\frac{\Lambda}{m}\right)^{N N_{f}} \int d \Phi d Q d \tilde{Q} e^{-W}
$$

where

$$
W=\tilde{Q}_{i}^{a} Q_{a}^{i}-\frac{\beta S}{2 N} \tilde{Q}_{i}^{a} Q_{a}^{j} \tilde{Q}_{j}^{b} Q_{b}^{i}, \quad \beta=\frac{g^{2}}{m M^{2}} .
$$

We will write the correlation function $<\tilde{Q}_{i}^{a} Q_{b}^{j}>$ as

$$
<\tilde{Q}_{i}^{a} Q_{b}^{j}>=\Delta^{\prime} \delta_{i}^{j} \delta_{b}^{a}
$$

Then the above tree level potential $W$ can be written in the form

$$
\begin{aligned}
W=\quad & \tilde{Q}_{i}^{a} Q_{a}^{i}-\beta S \Delta^{\prime} \tilde{Q}_{i}^{a} Q_{a}^{i}+N N_{f} \frac{\beta S}{2} \Delta^{\prime 2} \\
& \left.-\frac{\beta S}{2 N} \sum_{i, j=1}^{N_{f}}\left\{\sum_{a=1}^{N}\left(\tilde{Q}_{i}^{a} Q_{a}^{j}-\Delta^{\prime} \delta_{i}^{j}\right)\right]\left[\sum_{b=1}^{N}\left(\tilde{Q}_{j}^{b} Q_{b}^{i}-\Delta^{\prime} \delta_{j}^{i}\right)\right]\right\} .
\end{aligned}
$$

As a mean field theory, we neglect the contribution of the last term. Namely, we consider the following free energy:

$$
e^{-\frac{N}{S} \mathcal{F}}=\left(\frac{\Lambda}{m}\right)^{N N_{f}} \int d \Phi d Q d \tilde{Q} e^{-\left[\tilde{Q}_{i}^{a} Q_{a}^{i}-\beta S \Delta^{\prime} \tilde{Q}_{i}^{a} Q_{a}^{i}+N N_{f} \frac{\beta S}{2} \Delta^{\prime 2}\right]},
$$

We can compute the correlation function $<\tilde{Q}_{i}^{a} Q_{b}^{j}>=\Delta^{\prime} \delta_{i}^{j} \delta_{b}^{a}$ and get a consistency equation for $\Delta^{\prime}$ :

$$
\frac{1}{1-\beta S \Delta^{\prime}}=\Delta^{\prime} .
$$


This equation can be easily solved by

$$
\Delta^{\prime}=\frac{1-\sqrt{1-4 \beta S}}{2 \beta S}=\frac{2}{1+\sqrt{1-4 \beta S}} .
$$

Performing the Gaussian integral in (3.9), we get a free energy

$$
\begin{aligned}
\mathcal{F} & \left.=N_{f} S\left[\ln \frac{\Lambda}{m}-\ln \Delta^{\prime}+\frac{\beta S}{2} \Delta^{\prime 2}\right)\right] \\
& =N_{f} S\left[\ln \frac{\Lambda}{m}-\frac{1}{2}-\frac{1}{4 \beta S}(1-\sqrt{1-4 \beta S})+\ln \frac{1+\sqrt{1-4 \beta S}}{2}\right]
\end{aligned}
$$

which is exactly the result obtained by summing up the ladder diagrams 14 .

In general, we may have $N$ dependence in the self-consistency equation (3.10). Then, we should take the planar limit where $N$ dependence disappears. Then we expect that the result reflects the contribution from the planar diagrams.

\subsection{Effective Superpotential for Baryons from Matrix Theory}

Let us apply the mean-field technique to the baryonic perturbation. We consider the free energy

$$
e^{-\frac{N}{S} \mathcal{F}}=\left(\frac{N \Lambda}{S}\right)^{N} \int \prod_{i, a=1}^{N} d Q_{i}^{a} d \tilde{Q}_{a}^{i} e^{-\frac{N}{S} W_{t r e e}(Q, \tilde{Q})}
$$

which is in our case,

$$
\begin{aligned}
& e^{-\frac{N}{S} \mathcal{F}} \\
& =\left(\frac{N \Lambda}{S}\right)^{N} \int \prod_{i, a=1}^{N} d Q_{i}^{a} d \tilde{Q}_{a}^{i} e^{-\frac{N}{S}\left(m_{i} \tilde{Q}_{i}^{a} Q_{a}^{i}+b \epsilon^{a_{1} a_{2} \cdots a_{N}} Q_{a_{1}}^{1} Q_{a_{2}}^{2} \cdots Q_{a_{N}}^{N}+\tilde{b} \epsilon_{b_{1} b_{2} \cdots b_{N}} \tilde{Q}_{1}^{b_{1}} \tilde{Q}_{2}^{b_{2} \cdots \tilde{Q}_{N}} \tilde{b}_{N}\right)}
\end{aligned}
$$

The superpotential can be derived by the free energy $\mathcal{F}$ as

$$
W=N S\left(1-\ln \frac{S}{\Lambda^{3}}\right)+\mathcal{F}
$$

The first term of the superpotential is the Veneziano-Yankielowicz superpotential which can be obtained either from the volume of the gauge groups [12] or Gaussian integral measures [13. We will first re-scale the variables

$$
Q_{i}^{a} \rightarrow\left(\frac{N}{S} m_{i}\right)^{-1 / 2} Q_{i}^{a}, \quad \tilde{Q}_{a}^{i} \rightarrow\left(\frac{N}{S} m_{i}\right)^{-1 / 2} \tilde{Q}_{a}^{i} .
$$


Then the matrix integral can be written as

$$
\begin{aligned}
& e^{-\frac{N}{S} \mathcal{F}} \\
&\left(\frac{\Lambda^{N} m_{2} \cdots m_{n}}{m_{1}}\right)^{N} \int \prod_{i, a=1}^{N} d Q_{i}^{a} d \tilde{Q}_{a}^{i} e^{-W} .
\end{aligned}
$$

where

$$
\begin{aligned}
& W=\tilde{Q}_{i}^{a} Q_{a}^{i} \\
& -\left[\left(\frac{S}{N}\right)^{N-2} \frac{1}{m_{1} m_{2} \cdots m_{n}}\right]^{1 / 2}\left(b \epsilon^{a_{1} a_{2} \cdots a_{N}} Q_{a_{1}}^{1} Q_{a_{2}}^{2} \cdots Q_{a_{N}}^{N}+\tilde{b} \epsilon_{b_{1} b_{2} \cdots b_{N}} \tilde{Q}_{1}^{b_{1}} \tilde{Q}_{2}^{b_{2}} \cdots \tilde{Q}_{N}^{b_{N}}\right)
\end{aligned}
$$

Integrating out the fields $Q_{a}^{N}$ and $\tilde{Q}_{N}^{a}$, we get

$$
e^{-\frac{N}{S} \mathcal{F}}=\left(\frac{\Lambda^{N}}{m_{1} m_{2} \cdots m_{n}}\right)^{N} \int \prod_{i=1}^{N-1} \prod_{a=1}^{N} d Q_{i}^{a} d \tilde{Q}_{a}^{i} e^{-\tilde{W}}
$$

where $\tilde{W}$ is given by

$$
\tilde{W}=-\tilde{Q}_{i}^{a} Q_{a}^{i}+\frac{\alpha}{N^{N-2}} \epsilon^{a_{1} a_{2} \cdots a_{N}} \epsilon_{b_{1} b_{2} \cdots a_{N}} Q_{a_{1}}^{1} Q_{a_{2}}^{2} \cdots Q_{a_{N-1}}^{N-1} \tilde{Q}_{1}^{b_{1}} \tilde{Q}_{2}^{b_{2}} \cdots \tilde{Q}_{N-1}^{b_{N-1}}
$$

and the parameter $\alpha$ is defined in (2.14). To estimate the free energy, we apply the meanfield approximation. We put the correlation functions $<\epsilon^{a a_{1} a_{2} \cdots a_{N-1} a_{N}} \epsilon_{b_{1} b_{2} \cdots b_{N-1} a_{n}} \prod_{k \neq i}^{N-1} Q_{a_{i}}^{k} \tilde{Q}_{k}^{b_{i}}>$ in the form

$$
<\epsilon^{a a_{1} a_{2} \cdots a_{N-1} a_{N}} \epsilon_{b_{1} b_{2} \cdots b_{N-1} a_{n}} \prod_{k \neq i}^{N-1} Q_{a_{i}}^{k} \tilde{Q}_{k}^{b_{i}}>=(N-1) N^{N-2} \Delta^{\prime} \delta_{a_{i}}^{b_{i}} .
$$

Then we can rewrite $\tilde{W}$ as

$$
\begin{aligned}
& \tilde{W}=-\tilde{Q}_{i}^{a} Q_{a}^{i}+\alpha \Delta^{\prime} \tilde{Q}_{i}^{a} Q_{a}^{i}-\alpha \Delta^{\prime}<Q_{a}^{i} \tilde{Q}_{i}^{a}> \\
& +\sum_{i=1}^{N-1} \frac{\alpha \epsilon^{a_{1} a_{2} \cdots a_{N}} \epsilon_{b_{1} b_{2} \cdots a_{N}}}{(N-1) N^{N-2}} \prod_{k \neq i}^{N-1} Q_{a_{i}}^{k} \tilde{Q}_{k}^{b_{i}}<Q_{a_{i}}^{i} \tilde{Q}_{i}^{b_{i}}> \\
& +\alpha \sum_{i=1}^{N-1}\left(\frac{\epsilon^{a_{1} a_{2} \cdots a_{N}} \epsilon_{b_{1} b_{2} \cdots a_{N}}}{(N-1) N^{N-2}} \prod_{k \neq i}^{N-1} Q_{a_{i}}^{k} \tilde{Q}_{k}^{b_{i}}-\Delta^{\prime} \delta_{b_{i}}^{a_{i}}\right)\left(Q_{a_{i}}^{i} \tilde{Q}_{i}^{b_{i}}-<Q_{a_{i}}^{i} \tilde{Q}_{i}^{b_{i}}>\right)
\end{aligned}
$$

As a mean field evaluation, we neglect the contribution of the last term of equation (3.22). We also omit the contribution of the term

$$
\sum_{i=1}^{N-1} \frac{\alpha \epsilon^{a_{1} a_{2} \cdots a_{N}} \epsilon_{b_{1} b_{2} \cdots a_{N}}}{(N-1) N^{N-2}} \prod_{k \neq i}^{N-1} Q_{a_{i}}^{k} \tilde{Q}_{k}^{b_{i}}<Q_{a_{i}}^{i} \tilde{Q}_{i}^{b_{i}}>
$$


Note that the omitting of this term is not completely valid for $N=2$ and $N=3$. For $N=2$, this term is a constant and should be included in the free energy. This case, the integral in (3.19) can be performed without any approximation. We can easily see that the resulting expression of free energy agrees with the field theoretical results(2.16) for $N=2$. For $N=3$, (3.23) is bilinear in fields and we should prepare more generic ansatz for the correlator

$$
<\epsilon^{a a_{1} a_{2} \cdots a_{N-1} a_{N}} \epsilon_{b_{1} b_{2} \cdots b_{N-1} a_{n}} \prod_{k \neq i}^{N-1} Q_{a_{i}}^{k} \prod_{k \neq j} \tilde{Q}_{k}^{b_{i}}>=(N-1) N^{N-2} \Delta_{a_{i}, j}^{\prime b_{j}, i}
$$

to get a proper consistency equation. This ansatz is generic, and we should use for generic value of $N$. However, the solution of the self-consistency equation seems very difficult to be solved. We therefore, assume $N>3$ for later calculation and negrect these terms. Then we find that the remaining integrations are just Gaussian integrals so that we can get a self-consistent equation for $\Delta^{\prime}$ via the equation (3.21).

The gap equation of this model can be easily obtained as

$$
(N-1) !\left(\frac{1}{1-\alpha \Delta^{\prime}}\right)^{N-2}=(N-1) N^{N-2} \Delta^{\prime}
$$

which can be written as

$$
(1-2 / N)(1-3 / N) \cdots(1-(N-1) / N)\left(\frac{1}{1-\alpha \Delta^{\prime}}\right)^{N-2}=\Delta^{\prime}
$$

If we can take a very "naive" planar limit, we obtain the gap equation

$$
\left(\frac{1}{1-\alpha \Delta^{\prime}}\right)^{N-2}=\Delta^{\prime}
$$

We should admit that this "planar" limit is too subtle. This may be originated from a simple ansatz for the correlation functions (3.21). As a diagrammatic calculation, we should select only a small part of the diagrams to get this form of correlation functions. Therefore, we should use more generic ansatz (3.24) to perform a more rigorous analysis. However, the gap equation becomes very complicated. A partial evidence of the appearance of the gap equation (3.27) is the following. Instead of considering the more 
general ansatz, we enhance the number of diagrams we should correct by changing the correlation functions. Namely, we enhance the number by changing the equation as

$$
N<\prod_{k \neq i}^{N-1}\left(\sum_{a_{j}} Q_{a_{j}}^{k} \tilde{Q}_{k}^{a_{j}}\right)>=(N-1) N^{N-2} \Delta^{\prime} \delta_{a_{i}}^{b_{i}} .
$$

Since the correlation function can $<Q_{i}^{a} \tilde{Q}_{b}^{j}>$ can be given by

$$
<Q_{i}^{a} \tilde{Q}_{b}^{j}>=\frac{\delta_{i j} \delta_{a b}}{1-\alpha \Delta^{\prime}}
$$

we get a "gap equation" in the form

$$
\left(\frac{1}{1-\alpha \Delta^{\prime}}\right)^{N-2}=(1-1 / N) \Delta^{\prime}
$$

As a "planar" equation, this equation leads to (3.27).

In this paper, we assume the "planar" gap equation is given by (3.27). The reason we insist on the appearance of the gap equation will be given bellow. When we change variables to $\tilde{\Delta}$ by

$$
\frac{1}{1-\alpha \Delta^{\prime}}=1+\alpha \tilde{\Delta}
$$

we can easily find that equation (3.27) leads to

$$
\tilde{\Delta}=(1+\alpha \tilde{\Delta})^{N-1}
$$

which is exactly the equation (2.13). This strongly suggests that the exact planer limit of the gap equation is given by (3.27).

Let us evaluate the free energy within our approximation. Performing the Gaussian integrations, we can evaluate the free energy as

$$
\mathcal{F}=S \ln \frac{m_{1} m_{2} \cdots m_{N}}{\Lambda^{N}}+S(N-1) \ln \left(1-\alpha \Delta^{\prime}\right)+\alpha S(N-1) \Delta^{\prime} \frac{1}{1-\alpha \Delta^{\prime}} .
$$

We rewrite the free energy in terms of $\tilde{\Delta}$ by the identification (3.31) to obtain

$$
\mathcal{F}=S \ln \frac{m_{1} m_{2} \cdots m_{N}}{\Lambda^{N}}-S \ln \tilde{\Delta}+\alpha S(N-1) \tilde{\Delta} .
$$

This is the final form of the free energy which should be compared to the field theoretical result(2.16):

$$
\mathcal{F}=S \ln \frac{m_{1} m_{2} \cdots m_{N}}{\Lambda^{N}}-S \ln \tilde{\Delta}+\alpha S(N-2) \tilde{\Delta} .
$$

The difference of the last term may be related to our subtle planar limit. 


\section{Discussions}

We have discussed a method to obtain the effective superpotential for baryons and mesons by using the mean-field approximation. We have derived a result which is almost identicat to the field theoretical result. However, we have assumed "planar limit" of the self-consistency equation for the derivation. We could not prove the conjecture since we have used a simple ansatz for the correlation functions. We should use more rigorous ansatz to get an improved result. It seems interesting that the resulting expression from the field theory is of the form which may be fully obtained by the mean-field method. Our result may indicates the existence of some simple derivation of the superpotential for baryonic fields.

Another Interesting application of the mean-field method is for the gauge theories with $N_{f}>N_{c}$. In this case, the most interesting aspect is the appearance of Seiberg duality which has been treated in the framework of the matrix models in [29, 30, 21] However, the baryonic degree of freedom will be required for the complete derivation of the duality. Mean-field approach may be useful to get the duality map of the fields identification. 


\section{References}

[1] R. Dijkgraaf, C. Vafa, "Matrix Models, Topological Strings, and Supersymmetric Gauge Theories", Nucl. Phys. B644 (2002) 3-20, [arXiv:hep-th/0206255].

[2] R. Dijkgraaf, C. Vafa, "On Geometry and Matrix Models", Nucl. Phys. B644 (2002) 21-39, [arXiv:hep-th/0207106].

[3] R. Dijkgraaf and C. Vafa,, "A Perturbative Window into Non-Perturbative Physics", [arXiv:hep-th/0208048] .

[4] L. Chekhov and A. Mironov,, "Matrix models vs. Seiberg-Witten/Whitham theories", [arXiv:hep-th/0209085].

[5] N. Dorey, T. J. Hollowood, S. Prem Kumar and A. Sinkovics, Exact superpotentials from matrix models. [arXiv:hep-th/0209089]; Massive vacua of $\mathrm{N}=1^{*}$ theory and S-duality from matrix models [arXiv:hep-th/0209099]

[6] F. Ferrari, "On exact superpotentials in confining vacua", [arXiv:hep-th/0210135].

[7] H. Fuji and Y. Ookouchi, "Comments on effective superpotentials via matrix models", [arXiv:hep-th/0210148].

[8] D. Berenstein, "Quantum moduli spaces from matrix models", [arXiv:hepth/0210183].

[9] N. Dorey, T. J. Hollowood and S. P. Kumar, "S-duality of the Leigh-Strassler Deformation via Matrix Models", [arXiv:hep-th/0210239].

[10] H. Itoyama, A. Morozov, "The Dijkgraaf-Vafa prepotential in the context of general Seiberg-Witten theory", [arXiv:hep-th/0211245].

[11] H. Itoyama, A. Morozov, "Experiments with the WDVV equations for the gluinocondensate prepotential: the cubic (two-cut) case", [arXiv:hep-th/0211259]. 
[12] R.Dijkgraaf, M.T.Grisaru, C.S.Lam,C.Vafa, and D.Zanon, "Perturbative Computation of Glueball Superpotentials", [arXiv:hep-th/0211017].

[13] F. Cachazo, M. R. Douglas, N. Seiberg, E. Witten, "Chiral Rings and Anomalies in Supersymmetric Gauge Theory", [arXiv:hep-th/0211170].

[14] R. Argurio, V.Campos, G.Ferretti and R.Heise, "Exact Superpotentials for Theories with Flavors via Matrix Integral', [arXiv:hep-th/0210291].

[15] J. McGreevy, "Adding flavor to Dijkgraaf-Vafa", [arXiv:hep-th/0211009].

[16] H. Suzuki, "Perturbative Derivation of Exact Superpotential for Meson Fields from Matrix Theories with One Flavour", [arXiv:hep-th/0211052].

[17] I. Bena, R. Roiban, "Exact superpotentials in $N=1$ theories with flavor and their matrix model formulation", [arXiv:hep-th/0211075].

[18] Y. Demasure, R. A. Janik, "Effective matter superpotentials from Wishart random matrices", [arXiv:hep-th/0211082].

[19] Y.Tachikawa, "Derivation of the Konishi anomaly relation from Dijkgraaf-Vafa with (Bi-)fundamental matters", [arXiv:hep-th/0211189].

[20] Y. Ookouchi, "N=1 Gauge Theory with Flavor from Fluxes", [arXiv:hepth/0211287].

[21] K. Ohta, "Exact Mesonic Vacua From Matrix Moodels", [arXiv:hep-th/0212025].

[22] Y.Tachikawa, "Derivation of the linearity principle of Intriligator-Leigh-Seiberg", [arXiv:hep-th/0211274].

[23] R. Argurio,V.L. Campos,G. Ferretti,R. Heise, "Baryonic Corrections to Superpotentials from Perturbation Theory", [arXiv:hep-th/0211249].

[24] I. Bena, R. Roiban, R. Tatar, "Baryons, Boundaries and Matrix Models", [arXiv:hepth/0211271]. 
[25] G. Veneziano and S. Yankielowicz, "An Effective Lagrangian For The Pure N=1 Supersymmetric Yang-Mills Theory", Phys. Lett. B 113 (1982) 231.

[26] N. Seiberg, "Exact Results on the Space of Vacua of Four Dimensional SUSY Gauge Theories", Phys. Rev. D49 (1994) 6857-6863, [arXiv:hep-th/9402044].

[27] K. A. Intriligator and N. Seiberg, "Phases of N=1 supersymmetric gauge theories in four-dimensions", Nucl. Phys. B 431 (1994) 551 [arXiv:hep-th/9408155].

[28] K. A. Intriligator, R. G. Leigh and N. Seiberg, "Exact superpotentials in fourdimensions", Phys. Rev. D 50 (1994) 1092 [arXiv:hep-th/9403198].

[29] B. Feng, "Seiberg Duality in Matrix Model", [arXiv:hep-th/0211202].

[30] B. Feng, Y.-H. He, "Seiberg Duality in Matrix Models II", [arXiv:hep-th/0211234]. 